\title{
The mushroom Ganoderma lucidum suppresses breast-to-lung cancer metastasis through the inhibition of pro-invasive genes
}

\author{
JAGADISH LOGANATHAN $^{1 *}$, JIAHUA JIANG ${ }^{1 *}$, AMANDA SMITH $^{1}$, ANDREJ JEDINAK $^{1}$, \\ ANITA THYAGARAJAN-SAHU ${ }^{1}$, GEORGE E. SANDUSKY ${ }^{2}$, HARIKRISHNA NAKSHATRI ${ }^{3,4,6}$ and DANIEL SLIVA ${ }^{1,5}$ \\ ${ }^{1}$ Cancer Research Laboratory, Methodist Research Institute, Indiana University Health, Indianapolis, IN 46202; \\ Departments of ${ }^{2}$ Pathology, ${ }^{3}$ Biochemistry and Molecular Biology, ${ }^{4}$ Surgery and ${ }^{5}$ Medicine, ${ }^{6}$ Indiana University \\ Simon Cancer Center, Indiana University School of Medicine, Indianapolis, IN 46202, USA
}

Received January 15, 2014; Accepted February 26, 2014

DOI: 10.3892/ijo.2014.2375

\begin{abstract}
Breast cancer metastasis is one of the major reasons for the high morbidity and mortality of breast cancer patients. In spite of surgical interventions, chemotherapy, radiation therapy and targeted therapy, some patients are considering alternative therapies with herbal/natural products. In the present study, we evaluated a well-characterized extract from the medicinal mushroom Ganoderma lucidum (GLE) for its affects on tumor growth and breast-to-lung cancer metastasis. MDA-MB-231 human breast cancer cells were implanted into the mammary fat pads of nude mice. GLE (100 mg/kg/every other day) was administered to the mice by an oral gavage for 4 weeks, and tumor size was measured using microcalipers. Lung metastases were evaluated by hematoxylin and eosin (H\&E) staining. Gene expression in MDA-MB-231 cells was determined by DNA microarray analysis and confirmed by quantitative PCR. Identified genes were silenced by siRNA, and cell migration was determined in Boyden chambers and by wound-healing assay. Although an oral administration of GLE only slightly suppressed the growth of large tumors, the same treatment significantly inhibited the number of breast-to-lung cancer metastases. GLE also downregulated the expression of genes associated with invasive behavior (HRAS, VIL2, S100A4, MCAM, I2PP2A and FN1) in MDA-MB-231
\end{abstract}

Correspondence to: Dr Daniel Sliva, Cancer Research Laboratory, Methodist Research Institute, IU Health, 1800 N Capitol Ave, E504, Indianapolis, IN 46202, USA

E-mail: dsliva@iuhealth.org

*Contributed equally

Abbreviations: GLE, Ganoderma lucidum extract; VIL2, ezrin; FN1, fibronectin 1; MCAM, melanoma cell adhesion molecule; S100A4, S100 calcium binding protein A4; I2PP2A, SET nuclear oncogene; HRAS, v-Ha-Ras Harvey rat sarcoma viral oncogene homolog

Key words: mushroom, Ganoderma lucidum, polysaccharides, triterpenes, breast-to-lung cancer metastases, gene expression, cell migration cells. Gene silencing of HRAS, VIL2, S100A4, I2PP2A and FN1 by siRNA suppressed migration of MDA-MB-231 cells. Our study suggests that an oral administration of GLE can inhibit breast-to-lung cancer metastases through the downregulation of genes responsible for cell invasiveness. The anti-metastatic benefits of GLE warrant further clinical studies.

\section{Introduction}

Breast cancer is a leading cause of cancer death in women worldwide and the second leading cause of cancer death in the United States $(1,2)$. The high mortality among cancer patients is associated with cancer metastasis, which contributes to more than $90 \%$ of cancer-related fatalities (3). Although chemotherapy, radiation therapy and targeted therapy can directly kill cancer cells, some cancer cells are resistant to these treatments and can further proliferate and metastasize. Therefore, identifying new drugs/compounds with an anti-invasive potential would help to control the metastatic properties of cancer cells. Interestingly, some natural/dietary compounds show the potential to suppress proliferation and invasiveness of cancer cells (4).

One of the dietary compounds not widely consumed in the United States is the mushroom. However, two recent epidemiological studies from Asia suggest that mushrooms can actually protect against breast cancer (5,6). Ganoderma lucidum is a mushroom recognized by traditional Chinese medicine (TCM) and commonly used in the forms of tea, powder and dietary supplements (7). The botanical characterization, description and therapeutic effects of G. lucidum are summarized in the American Herbal Pharmacopoeia (Reishi Mushroom; www.herbal-ahp.org). We have previously shown that G. lucidum extract (GLE) containing triterpenes and polysaccharides, suppresses the invasive behavior of breast cancer cells $(8,9)$. Experimental in vivo studies demonstrated the inhibition of liver and lung metastases of lung carcinoma cells by triterpenoid fraction of G. lucidum and isolated ganoderic acid Me (GA-Me) and T (GA-T), respectively (10-12). In addition, $2.5 \%$ of the antlered form of G. lucidum in the diet suppressed the number of lung metastases of lung cancer cells (13). Oral administration of a lucidenic acid-rich 
G. lucidum extract inhibited lung and liver metastases of human hepatoma cells in a xenograft model (14).

In the present study, we evaluated the effect of GLE on the growth and breast-to-lung cancer metastasis in an orthotopic xenograft model without significantly influencing primary tumor growth. Our data suggest that an oral application of GLE inhibits lung metastases and can be used for the natural/alternative therapy of invasive breast cancers.

\section{Materials and methods}

Materials. Human breast cancer cells (MDA-MB-231) were obtained from ATCC (Manassas, VA). MDA-MB-231 cells were maintained in DMEM medium supplemented with penicillin $(50 \mathrm{U} / \mathrm{ml})$, streptomycin $(50 \mathrm{U} / \mathrm{ml})$, and $10 \%$ fetal bovine serum (FBS). Media and supplements were from Invitrogen (Grand Island, NY). FBS was obtained from Hyclone (Logan, UT). GLE was supplied by Pharmanex (Provo, UT). GLE is a standardized Ganoderma lucidum extract containing $6 \%$ triterpenes and $13.5 \%$ polysaccharides; the extraction procedure was previously described (15). GLE stock solution was prepared in water for animal experiments or in DMSO for cell culture experiments. siRNA reagents, scrambled siRNA and SiRNA for HRAS, VIL2, S100A4, MCAM, I2PP2A, and FN1 were from Santa Cruz Biotechnology (Santa Cruz, CA).

Human breast tumor xenograft experiments. MDA-MB-231 cells $\left(1 \times 10^{6}\right)$ in $0.2 \mathrm{ml}$ DMEM were injected into the mammary fat pad of 6- to 7-week-old female nude mice (Harlan, Indianapolis, IN), as previously described (16). Three to four weeks after implantation with tumor cells, when tumors reached approximately $600 \mathrm{~mm}^{3}$, the animals were randomized into control and treatment groups (18 animals per group). The animals received intragastrical gavage every other day with water (control) or $100 \mathrm{mg} \mathrm{GLE} / \mathrm{kg}$ of body weight (treatment) for an additional 28 days. The tumor size was measured using calipers, and the tumor volume was estimated by the formula: tumor volume $\left(\mathrm{mm}^{3}\right)=\mathrm{W}^{2} \times \mathrm{L} \times 1 / 2$, where $\mathrm{L}$ is the length and $\mathrm{W}$ is the width of the tumor. At the end of the experiment (day 28), the lungs were harvested and fixed in $10 \%$ neutral buffered formalin at $4^{\circ} \mathrm{C}$ for $24 \mathrm{~h}$. Tissue was then processed overnight and embedded in paraffin. Five-micrometer sections were stained with hematoxylin and eosin $(\mathrm{H} \& \mathrm{E})$, and the metastases in whole sections of stained lungs from 6 animals in each of the control and GLE-treatment groups were evaluated under a light microscope by 3 independent observers. The protocol for animal experiments was approved by the Animal Research Committee at IU Health Methodist Hospital according to the NIH guidelines for the Care and Use of Laboratory Animals.

DNA microarrays. MDA-MB-231 cells were treated with GLE (0 and $1.0 \mathrm{mg} / \mathrm{ml}$ ) for $24 \mathrm{~h}$ and total RNA was isolated with RNAeasy (Qiagen, Valencia, CA). This RNA was used for the evaluation of gene expression with Oligo GEArray Human Tumor Metastasis Microarray according to the manufacturer's protocol (SA Biosciences, Frederick, MD, USA).

Quantitative RT-PCR. The quantitative real-time polymerase chain reaction (qRT-PCR) was performed using the ABI Prism 7900HT Fast Real-Time PCR System (Applied Biosystems,
Foster City, CA) according to the manufacturer's instructions. MDA-MB-231 cells were treated with GLE $(0$ and $1.0 \mathrm{mg} / \mathrm{ml})$ for $24 \mathrm{~h}$ and total RNA was isolated using RNAeasy (Qiagen). The RNA samples were reverse transcribed into cDNA (RT-PCR) using random hexamer primers and the TaqMan reverse transcription kit (Applied Biosystems). The cDNA (100 ng per sample) was subjected to qPCR analysis in quadruplicate using forward and reverse primers, the TaqMan Universal Master Mix, and a probe (10 $\mu$ l per reaction) in fast optical 96-well plates. The data were analyzed using the ABI Prism 7900 relative quantification $(\triangle \Delta \mathrm{Ct})$ study software (Applied Biosystems). We used primers for HRAS, VIL2, S100A4, MCAM, I2PP2A and $F N 1$ genes with the $\beta$-actin gene as the internal control (Applied Biosystems). The gene expressions levels were normalized to $\beta$-actin and are presented as arbitrary fold changes compared between the control and GLE-treated cells.

siRNA experiments. MDA-MB-231 cells $\left(2 \times 10^{5}\right)$ were seeded into 6-well plates and incubated at $37^{\circ} \mathrm{C}$ in a $5 \% \mathrm{CO}_{2}$ incubator until 70-80\% confluent. The cells were transfected with control RNA (scrambled, scRNA) or siRNA according to the manufacturer's protocol (Santa Cruz Biotechnology). Gene silencing by siRNA in MDA-MB-231 cells was evaluated by western blot analysis.

Western blot analysis. MDA-MB-231 cells were treated with GLE ( 0 and $1.0 \mathrm{mg} / \mathrm{ml}$ ) for $24 \mathrm{~h}$. Whole cell extracts were isolated as described (15), membrane extracts were isolated by using a ProteoExtract ${ }^{\circledR}$ subcellular proteome extraction kit (Merck, Darmstadt, Germany) according to the manufacturer's protocol. Protein expression was detected by western blot analysis with the corresponding antibodies anti-HRAS, anti-ezrin, anti-S100A4, anti-MCAM, anti-SET, anti-fibronectin, and anti- $\beta$-actin, anti-GAPDH, and anti- $\alpha$-integrin 3 as loading controls (Santa Cruz Biotechnology) as previously described (15). Reactive bands were visualized with a respective secondary antibody via an enhanced chemiluminescence (ECL) detection system.

Cell migration assay. The effect of gene silencing on cell migration of MDA-MB-231 cells was assessed in Boyden chambers as previously described (17). After fixing and staining, the number of migrating cells was counted from at least four random fields using a microscope at x20 magnification (17). Data points represent the average SD of individual filters within one representative experiment repeated at least twice.

Wound healing assay. MDA-MB-231 cells were untransfected (control) or transfected with scRNA or siRNAs. After $24 \mathrm{~h}$ the cells were scratched using a $200-\mu 1$ pipette tip and further incubated for an additional $24 \mathrm{~h}$. The extent of wound healing was observed microscopically and recorded.

Statistical analysis. Data are represented as mean \pm SD and were analyzed using SigmaPlot 11.2 (Systat Software Inc, San Jose, CA, USA).

\section{Results and Discussion}

Although previous studies demonstrated the suppression of tumor growth and the inhibition of metastases by purified 


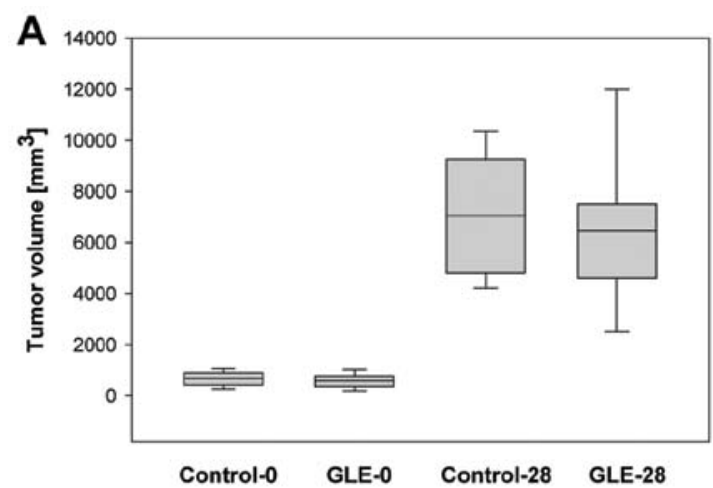

C

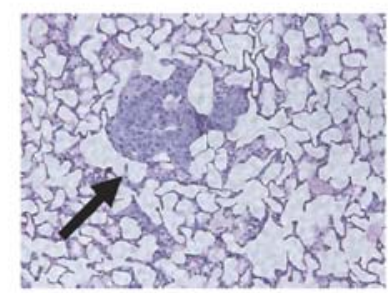

Control

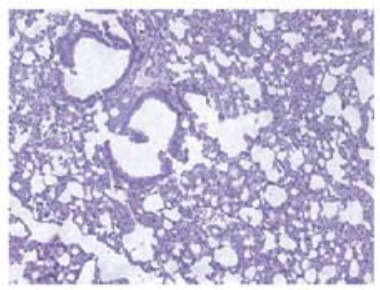

GLE
B

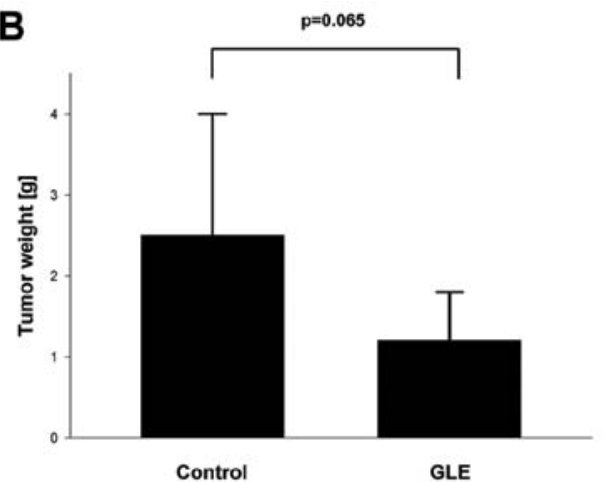

D

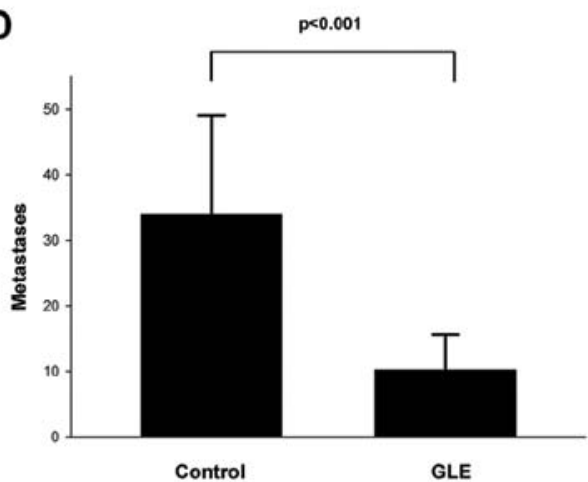

Figure 1. Effect of GLE on tumor growth and breast-to-lung cancer metastases. MDA-MB-231 breast cancer cells were implanted in the mammary fat pads of nude mice and treated with GLE as described in Materials and methods. (A) The size and (B) weight of tumors were measured after 28 days (n=16-18). (C) Representative H\&E stained lungs from control and GLE-treated groups; black arrows indicate metastasis. (D) Lung metastases were quantified as described in Materials and methods $(\mathrm{P}<0.001, \mathrm{n}=6)$.

Ganoderma lucidum compounds or extracts in experimental animals, these studies usually started the treatment with small tumors close to $100 \mathrm{~mm}^{3}$ in size $(12,14)$. Since not all breast cancers are diagnosed in the early stages, we were interested to learn whether GLE inhibits the growth and metastases of larger tumors. Highly invasive human breast cancer cells MDA-MB-231 were injected into the mammary fat pads of mice, and an oral application of GLE (100 mg/kg/body weight every other day) was started when the tumors reached $600 \mathrm{~mm}^{3}$. GLE treatment for 4 weeks had modest inhibitory effects on tumor size and weight (Fig. 1). Since we have previously shown that GLE inhibits invasive behavior in MCF-7 and MDA-MB-231 breast cancer cells in vitro $(8,9)$, we further studied whether GLE inhibits breast-to-lung cancer metastases in vivo. Although we did not observe changes in the tumor volumes in the control and GLE-treatment groups in MDA-MB-231 cell-derived tumors (Fig. 1A), we found statistically non-significant inhibition of tumor growth by GLE (Fig. 1B). This effect was caused by the necrosis since both control and GLE-treated tumors had necrotic central regions that were filled with fluid. However, our data show significant inhibition of breast-to-lung cancer metas-

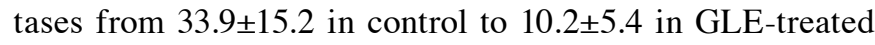
animals $(\mathrm{P}<0.001)$ (Fig. 1C and D).

In order to identify which pro-metastatic genes are affected in MDA-MB-231 cells by GLE, MDA-MB-231 cells were treated with vehicle or GLE $(24 \mathrm{~h}, 1.0 \mathrm{mg} / \mathrm{ml})$ and gene expression was analyzed by Oligo GEArray Human Tumor

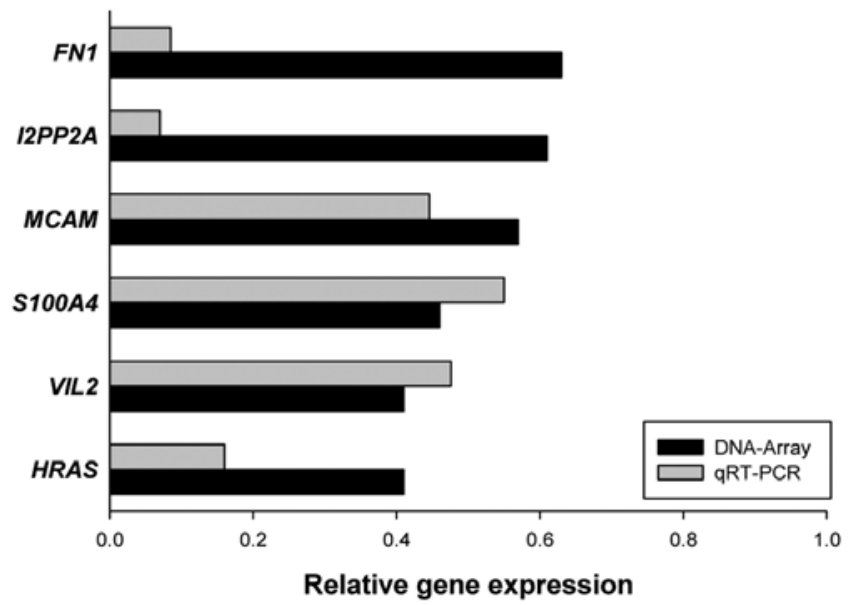

Figure 2. Effect of GLE on human tumor metastasis genes. MDA-MB-231 cells were treated with vehicle or GLE for $24 \mathrm{~h}$ and the gene expression was determined by Oligo GEArray Human Tumor Metastasis Microarray and confirmed by qRT-PCR as described in Materials and methods. The data are averages from 2 (microarray) and 4 (qRT-PCR) experiments. The fold change is relative to vehicle-treated cells

Metastasis Microarray as described in Materials and methods. GLE treatment downregulated the expression of HRAS, VIL2, S100A4, MCAM, I2PP2A and FN1 genes by more than $20 \%$, which we further confirmed by qRT-PCR (Fig. 2). 

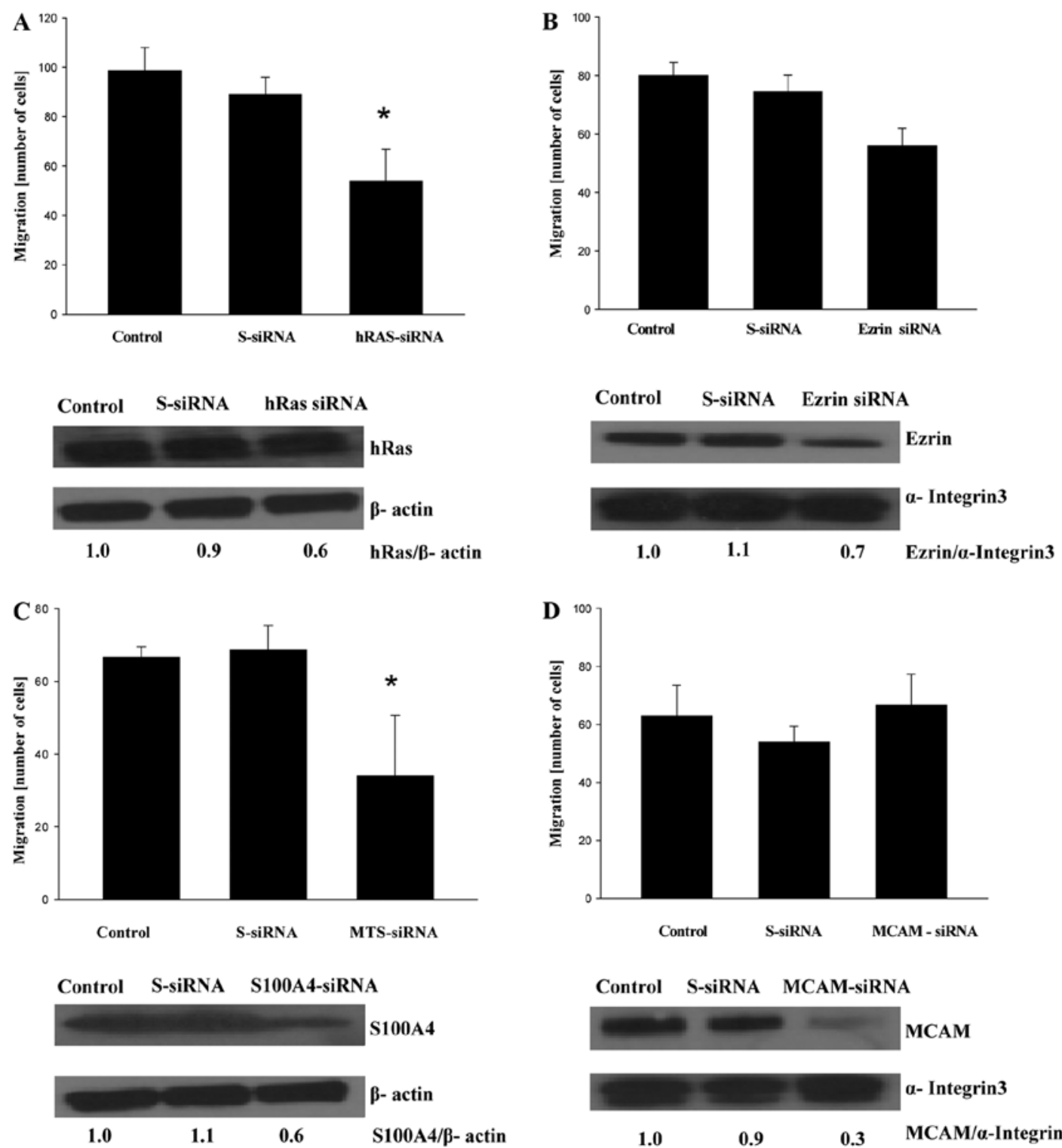

Control S-siRNA MCAM-siRNA
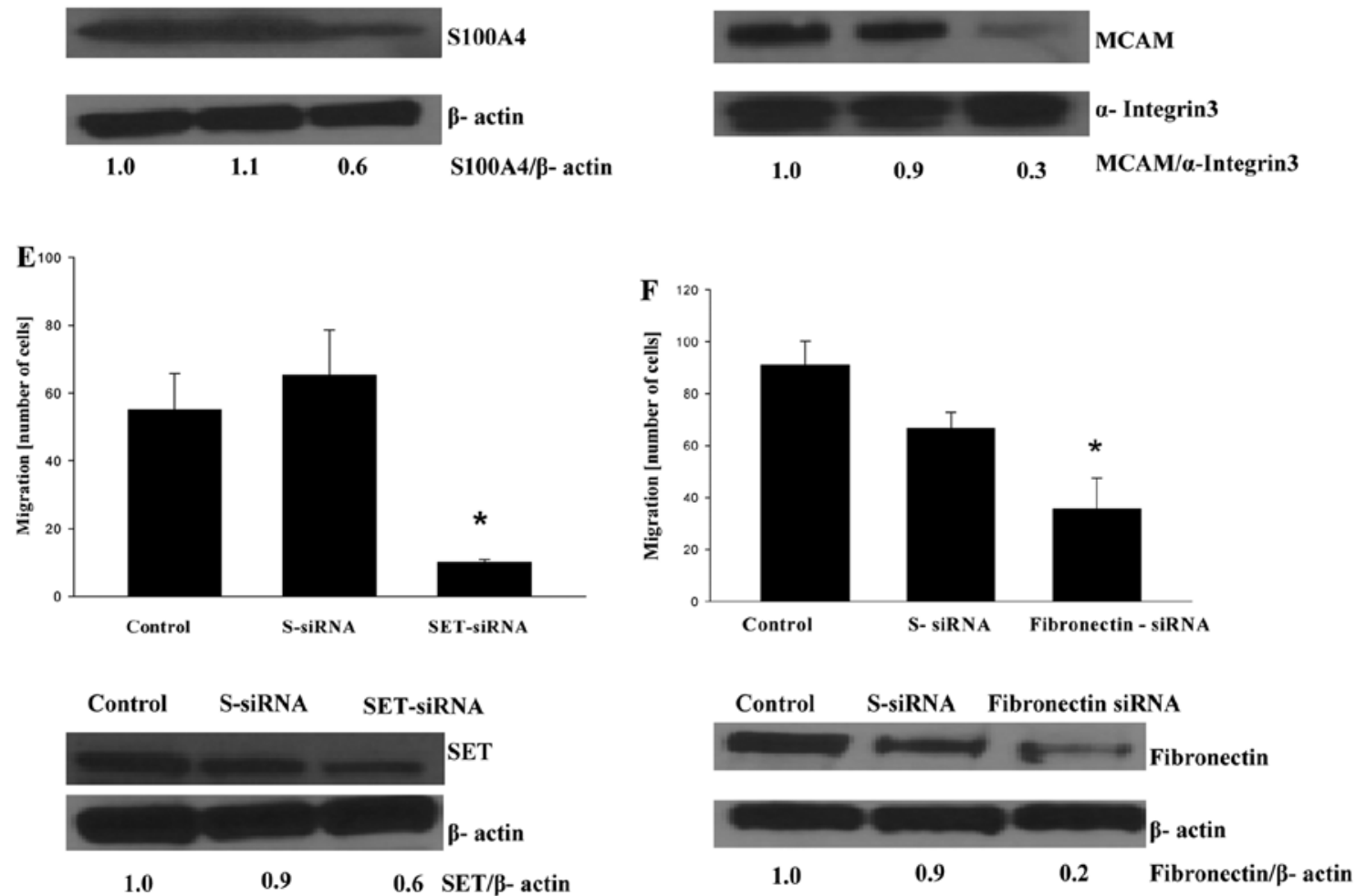

Figure 3. Effect of genetic silencing of GLE downregulated genes on cell migration. MDA-MB-231 cells were untransfected (control) or transfected with scrambled (sc-siRNA) or specific gene siRNA and cell migration and proper gene silencing was evaluated as described in Materials and methods. (A) HRAS, (B) VIL2 (ezrin), (C) S100A4, (D) MCAM, (E) I2PP2A (SET), (F) FN1 (fibronectin). The data are mean \pm SD (n=3), P $\leq 0.05$ by ANOVA. Western blots show representative experiment. 
A
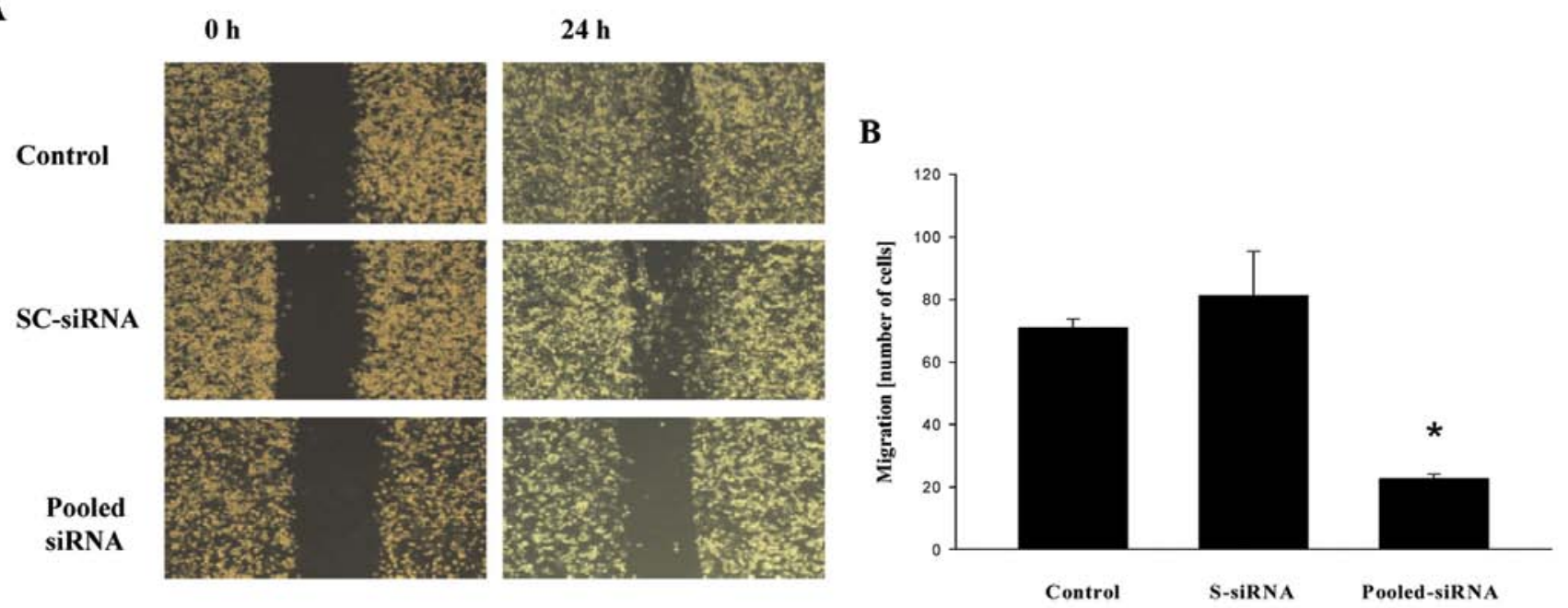

C
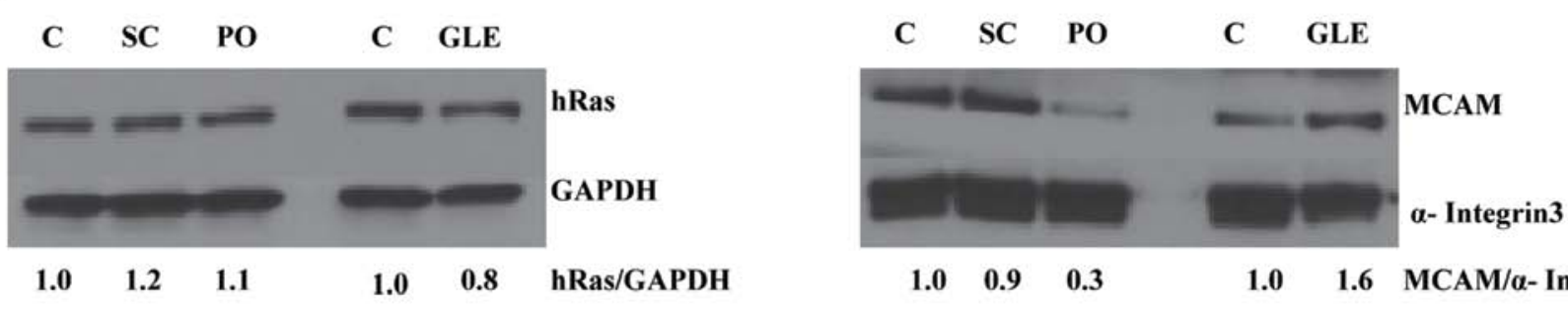

$\begin{array}{llllll}1.0 & 0.9 & 0.3 & 1.0 & 1.6 & \text { MCAM/ } \alpha-\text { Integrin } 3\end{array}$

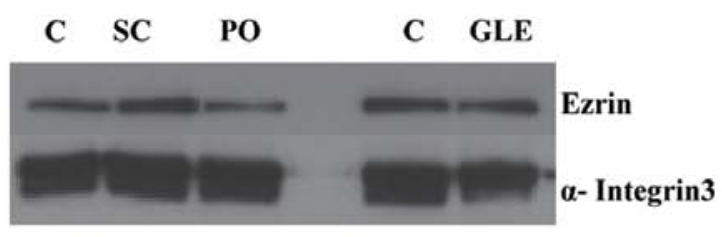

$\begin{array}{lll}1.0 & 1.2 & 0.7\end{array}$

$1.0 \quad 0.9 \quad$ Ezrin/a- Integrin 3
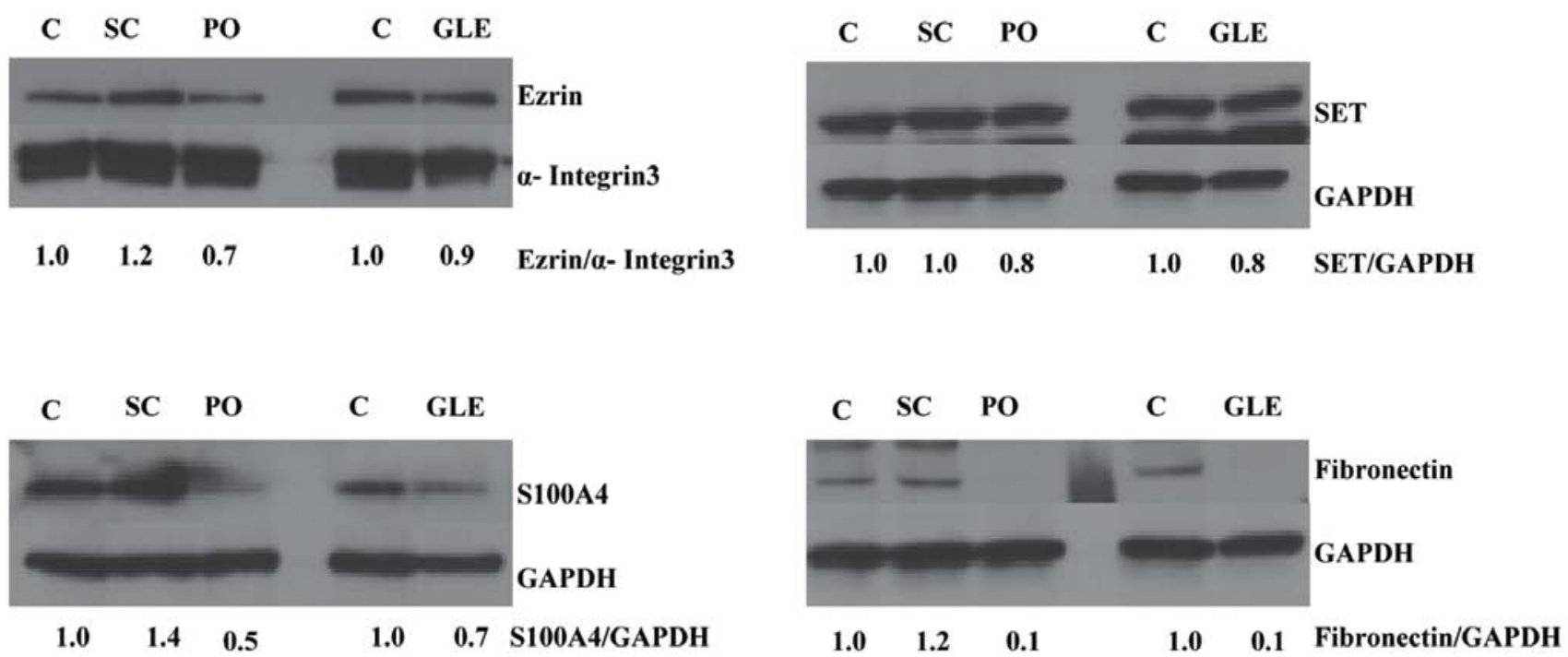

Figure 4. Pooled siRNA inhibits cell migration. MDA-MB-231 cells were untransfected (control) or transfected with scrambled (sc-siRNA) or a pool of siRNAs, (pooled-siRNA, containing HRAS-siRNA, VIL2-siRNA, S100A4-siRNA, MCAM-siRNA, I2PP2A-siRNA, and FN1-siRNA). Cell migration was evaluated by (A) the wound healing assay and (B) cell migration assay described in Materials and methods. The data are mean \pm SD ( $n=3$ ), $p \leq 0.05$ by $A N O V A$. (C) Representative western blots from MDA-MB-231 cells transfected with scrambled siRNA (SC) or pooled-siRNA (PO), or treated with GLE (GLE) or control (C).

To confirm that genes targeted by GLE are indeed responsible for the invasiveness of MDA-MB-231 cells, we silenced these genes by siRNA to evaluate whether this genetic manipulation suppressed the migration of MDA-MB-231 cells. Increased expression of the oncogene $H R A S$ is associated with aggressive breast cancer, and an overexpression of HRAS induces cell migration and an invasive phenotype in breast epithelial cells (18). Not surprisingly, HRAS silencing suppressed the migration of MDA-MB-231 cells (Fig. 3A). In agreement with a previous study by Li et al (19) on the silencing of VIL2, coding ezrin (VIL2), a cytoplasmic peripheral membrane protein that plays a key role in cell motility, slightly suppressed the migration of MDA-MB-231 cells (Fig. 3B). The S100A4 protein is overexpressed in highly metastatic cancers and controls cell migration through different pathways (20). Gene silencing of S100A4 also suppressed the migration of MDA-MB-231 cells (Fig. 3C). At 
the time of our manuscript preparation, Wang et al (21) demonstrated that gene silencing of S100A4 inhibited cell migration and invasion as well as lung metastases of MDA-MB-231 cells in mice. Although the original studies suggested that $M C A M$ (also known as CD146 membrane glycoprotein) is a tumor suppressor in breast carcinomas (22), CD146 expression was later associated with a poor prognosis in breast cancer patients and increased motility of breast cancer cells $(23,24)$. However, gene silencing of $M C A M$ did not affect the migration of MDA-MB-231 cells (Fig. 3D). The SET protein (gene I2PP2A) inhibits protein phosphatase $2 \mathrm{~A}$ (PP2A), which regulates oncoproteins (e.g. c-Myc, Bcr-Abl) in various cancers $(25,26)$. Therefore, the inhibition of $I 2 P P 2 A$ is therapeutically important, and, as recently demonstrated, targeting SET suppresses lung tumors (27). As seen in Fig. 3E, gene silencing of $I 2 P P 2 A$ also suppressed SET protein expression and the inhibited migration of MDA-MB-231 cells. The fibronectin 1 protein (gene FN1) controls cell adhesion and migration, and FN1 overexpression was detected in breast cancer metastases $(28,29)$. Gene silencing of FN1 resulted in the downregulation of FN1 expression and inhibited the migration of MDA-MB-231 cells (Fig. 3F). Since cell migration is a complex process and is controlled by more than one protein, we evaluated whether the gene silencing of all genes whose expression was downregulated by GLE treatment inhibited cell migration to a larger extent than the silencing of these genes individually. MDA-MB-231 cells were transfected with a mixture of siRNAs for HRAS, VIL2, S100A4, MCAM, $I 2 P P 2 A$ and $F N 1$, and cell migration was evaluated. As seen in Fig. 4A, transfection of pooled siRNAs suppressed migration in the wound-healing assay as well as in the cell migration assay in Boyden chambers (Fig. 4B), suggesting that targeting a pool of pro-invasive genes is a better strategy than targeting only one gene. On the other hand, the expression of ezrin, S100A4, MCAM, SET and fibronectin was downregulated by pooled siRNA, whereas the expression of HRAS was not affected (Fig. 4C). In addition, pooled siRNA and GLE treatment demonstrated the strongest inhibition of fibronectin expression, suggesting that fibronectin is the major target for inhibiting cell invasiveness. Although our data with pooled siRNA generally confirms our previous results with the isolated siRNA (Fig. 3), it is possible that pooled siRNA could also inhibit some of the single siRNA since HRAS-siRNA downregulated expression of the HRAS protein, whereas pooled siRNA does not. In addition, GLE treatment suppressed expression of different pro-invasive proteins with different potency, further suggesting specific targeting at transcriptional or posttranslational levels. These questions will be addressed in our future studies.

In our present study, we found that GLE downregulates the expression of a set of genes (HRAS, VIL2, S100A4, MCAM, $I 2 P P 2 A$ and $F N 1$ ) that are different from the previously published genes that mediate breast-to-lung cancer metastasis (30). One of the reasons for this difference is that in our experiments we originally evaluated the effect of GLE on the expression of selected pro-metastatic genes by using Oligo GEArray, which does not cover all genes. Moreover, the breast-to-lung metastatic genes identified by Minn et al (30) were overexpressed in the lung-metastatic derivative of MDA-MB-231 but not in the parental MDA-MB-231 cells used in our study. Therefore, a different set of genes should be targeted during the progression of metastatic breast cancer. In addition, our study was performed with only one cell line of highly metastatic triple negative breast cancer cells, MDA-MB-231 and different genes can be targeted in other metastatic breast cancers.

In conclusion, the chemically characterized dietary mushroom extract GLE inhibits breast-to-lung cancer metastasis of highly invasive human breast cancer cells implanted in mouse mammary tissue. In addition, GLE suppresses the expression of genes involved in the invasive behavior of cancer cells. Further preclinical studies evaluating GLE activity in the prevention of breast cancer metastasis are warranted.

\section{Acknowledgements}

This study was supported by the Methodist Research Institute, Indiana University Health, Indianapolis, IN, USA. We thank Ms. Elaine Bammerlin for the editing.

\section{References}

1. Jemal A, Bray F, Center MM, Ferlay J, Ward E and Forman D: Global cancer statistics. CA Cancer J Clin 61: 69-90, 2011.

2. Jemal A, Siegel R, Xu J and Ward E: Cancer statistics, 2010. CA Cancer J Clin 60: 277-300, 2010.

3. Christofori G: New signals from the invasive front. Nature 441: 444-450, 2006

4. Gullett NP, Ruhul Amin AR, Bayraktar S, et al: Cancer prevention with natural compounds. Semin Oncol 37: 258-281, 2010.

5. Zhang M, Huang J, Xie X and Holman CD: Dietary intakes of mushrooms and green tea combine to reduce the risk of breast cancer in Chinese women. Int J Cancer 124: 1404-1408, 2009.

6. Shin A, Kim J, Lim SY, Kim G, Sung MK, Lee ES and Ro J: Dietary mushroom intake and the risk of breast cancer based on hormone receptor status. Nutr Cancer 62: 476-483, 2010.

7. Wasser S: Reishi or Ling Zhi (Ganoderma lucidum). In: Encyclopedia of Dietary Supplements. Coates PM, Blackman MR, Cragg GM, Levine M, Moss J and White JD (eds). Marcel Dekker, New York, NY, pp603-622, 2005.

8. Thyagarajan A, Jiang J, Hopf A, Adamec J and Sliva D: Inhibition of oxidative stress-induced invasiveness of cancer cells by Ganoderma lucidum is mediated through the suppression of interleukin-8 secretion. Int J Mol Med 18: 657-664, 2006.

9. Thyagarajan A, Zhu J and Sliva D: Combined effect of green tea and Ganoderma lucidum on invasive behavior of breast cancer cells. Int J Oncol 30: 963-969, 2007.

10. Kimura Y, Taniguchi $M$ and Baba K: Antitumor and antimetastatic effects on liver of triterpenoid fractions of Ganoderma lucidum: mechanism of action and isolation of an active substance. Anticancer Res 22: 3309-3318, 2002.

11. Wang G, Zhao J, Liu J, Huang Y, Zhong JJ and Tang W: Enhancement of IL-2 and IFN-gamma expression and NK cells activity involved in the anti-tumor effect of ganoderic acid Me in vivo. Int Immunopharmacol 7: 864-870, 2007.

12. Chen NH, Liu JW and Zhong JJ: Ganoderic acid T inhibits tumor invasion in vitro and in vivo through inhibition of MMP expression. Pharmacol Rep 62: 150-163, 2010.

13. Nonaka Y, Ishibashi H, Nakai M, Shibata H, Kiso Y and Abe S: Effects of the antlered form of Ganoderma lucidum on tumor growth and metastasis in cyclophosphamide-treated mice. Biosci Biotechnol Biochem 72: 1399-1408, 2008.

14. Weng CJ, Chau CF, Yen GC, Liao JW, Chen DH and Chen KD: Inhibitory effects of Ganoderma lucidum on tumorigenesis and metastasis of human hepatoma cells in cells and animal models. J Agric Food Chem 57: 5049-5057, 2009.

15. Dudhgaonkar S, Thyagarajan A and Sliva D: Suppression of the inflammatory response by triterpenes isolated from the mushroom Ganoderma lucidum. Int Immunopharmacol 9: 1272-1280, 2009.

16. Sweeney CJ, Mehrotra S, Sadaria MR, et al: The sesquiterpene lactone parthenolide in combination with docetaxel reduces metastasis and improves survival in a xenograft model of breast cancer. Mol Cancer Ther 4: 1004-1012, 2005.

17. Sliva D. Mason R, Xiao H and English D: Enhancement of the migration of metastatic human breast cancer cells by phosphatidic acid. Biochem Biophys Res Commun 268: 471-479, 2000. 
18. Moon A, Kim MS, Kim TG, Kim SH, Kim HE, Chen YQ and Kim HR: H-ras, But not N-ras, induces an invasive phenotype in human breast epithelial cells: a role for MMP-2 in the H-ras-induced invasive phenotype. Int J Cancer 85: 176-181, 2000.

19. Li Q, Wu M, Wang H, Xu G, et al: Ezrin silencing by small hairpin RNA reverses metastatic behaviors of human breast cancer cells. Cancer Lett 261: 55-63, 2008.

20. Wang $Z$ and Griffin $M$ : The role of TG2 in regulating S100A4-mediated mammary tumour cell migration. PLoS One 8 e57017, 2013

21. Wang L, Wang X, Liang Y, Diao X and Chen Q: S100A4 promotes invasion and angiogenesis in breast cancer MDA-MB-231 cells by upregulating matrix metalloproteinase-13. Acta Biochim Pol 59: 593-598, 2012.

22. Shih IM: The role of CD146 (Mel-CAM) in biology and pathology. J Pathol 189: 4-11, 1999.

23. Zabouo G, Imbert AM, Jacquemier J, et al: CD146 expression is associated with a poor prognosis in human breast tumors and with enhanced motility in breast cancer cell lines. Breast Cancer Res 11: R1, 2009.

24. Zeng GF, Cai SX and Wu GJ: Up-regulation of METCAM/MUC18 promotes motility, invasion, and tumorigenesis of human breast cancer cells. BMC Cancer 11: 113, 2011.
25. Yeh E, Cunningham M, Arnold $\mathrm{H}$, et al: A signalling pathway controlling c-Myc degradation that impacts oncogenic transformation of human cells. Nat Cell Biol 6: 308-318, 2004

26. Salas A, Ponnusamy S, Senkal CE, et al: Sphingosine kinase-1 and sphingosine-1 phosphate receptor 2 mediate Bcr-Abll stability and drug resistance by modulation of protein phosphatase 2A. Blood 117: 5941-5952, 2011.

27. Saddoughi SA, Gencer S, Peterson YK, et al: Sphingosine analogue drug FTY720 targets I2PP2A/SET and mediates lung tumour suppression via activation of PP2A-RIPK1-dependent necroptosis. EMBO Mol Med 5: 105-121, 2013.

28. Pankov R and Yamada KM: Fibronectin at a glance. J Cell Sci 115: 3861-3863, 2002

29. Soikkeli J, Podlasz P, Yin M, et al: Metastatic outgrowth encompasses COL-I, FN1, and POSTN up-regulation and assembly to fibrillar networks regulating cell adhesion, migration, and growth. Am J Pathol 177: 387-403, 2010.

30. Minn AJ, Gupta GP, Siegel PM, et al: Genes that mediate breast cancer metastasis to lung. Nature 436: 518-524, 2005. 